\section{THE STRUCTURE OF CYCLOOCTATIN, A NEW INHIBITOR OF LYSOPHOSPHOLIPASE}

Sir:

In the preceding paper ${ }^{1)}$, we have described the taxonomy, isolation, physico-chemical properties and biological activities of cyclooctatin (Fig. 1), a novel inhibitor of lysophospholipase (Lyso-PL). In this paper, we describe the structure determination of cyclooctatin.

The molecular weight and formula of cyclooctatin were elucidated as $\mathrm{C}_{20} \mathrm{H}_{34} \mathrm{O}_{3}$ (MW 322.49) by the FD-MS peak at $m / z 322\left(\mathrm{M}^{+}\right)$, elemental analysis (found: $\mathrm{C} 74.09, \mathrm{H}$ 10.63; calcd for $\mathrm{C}_{20} \mathrm{H}_{34} \mathrm{O}_{3}$ : $\mathrm{C} 74.45, \mathrm{H} 10.63$ ) and ${ }^{1} \mathrm{H}$ and ${ }^{13} \mathrm{C}$ NMR spectra (Table 1). UV spectrum showed the end absorption in EtOH. IR spectrum $(\mathrm{KBr})$ showed the presence of hydroxy group $\left(3430 \mathrm{~cm}^{-1}\right) .{ }^{13} \mathrm{C}$ NMR spectrum revealed two signals of $s p^{2}$ carbon $\left(\delta_{\mathrm{C}} 119.1\right.$ and $154.5 \mathrm{ppm}$ ) and eighteen signals of $s p^{3}$ carbon in which three signals (C-3, C-4 and C-11) appeared in lower field $\left(\delta_{\mathrm{C}} 75.7,78.4\right.$ and $\left.63.4 \mathrm{ppm}\right)$ indicating the oxygen-bearing carbons.

In the ${ }^{1} \mathrm{H}-{ }^{1} \mathrm{H}$ COSY spectrum and proton decoupling NMR experiments, a vicinal-spin spin coupling between the signals at $\delta_{\mathrm{H}} 1.91,2.74\left(5-\mathrm{H}_{2}\right)$ ppm and the signal at $\delta_{\mathrm{H}} 5.28(6-\mathrm{H}) \mathrm{ppm}$ was observed, and vicinal-spin spin couplings between the signals at $\delta_{\mathrm{H}} 1.42,1.59\left(9-\mathrm{H}_{2}\right) \mathrm{ppm}$ and the signals at $\delta_{\mathrm{H}} 1.38,1.56\left(8-\mathrm{H}_{2}\right) \mathrm{ppm}, \delta_{\mathrm{H}} 1.38,1.56$ $\left(8-\mathrm{H}_{2}\right)$ and $2.30(7-\mathrm{H}) \mathrm{ppm}, \delta_{\mathrm{H}} 2.30(7-\mathrm{H})$ and 1.83 (13-H) ppm, $\delta_{\mathrm{H}} 1.83(13-\mathrm{H})$ and $0.79\left(15-\mathrm{H}_{3}\right) \mathrm{ppm}$, $\delta_{\mathrm{H}} 1.83(13-\mathrm{H})$ and $0.96\left(16-\mathrm{H}_{3}\right)$ ppm were also observed. Furthermore, vicinal-spin spin couplings indicated the linkage of $\mathrm{C}-10$ to $\mathrm{C}-11$ in the same fashion as described above. From the above results, the presence of three partial structures (Fig. 2A, B and C) were revealed.

As shown in Fig. 3, in the HMBC (heteronuclear multiple bond connectivity) spectrum, partial structures A, B and C could be connected as follows. The olefinic proton at $\delta_{\mathrm{H}} 5.28(6-\mathrm{H}) \mathrm{ppm}$ coupled to two carbons at $\delta_{\mathrm{C}} 55.1(\mathrm{C}-7)$ and $45.9(\mathrm{C}-9 \mathrm{a}) \mathrm{ppm}$, and the methine proton at $\delta_{\mathrm{H}} 2.30(7-\mathrm{H})$ ppm coupled to the carbon at $\delta_{\mathrm{C}} 154.5(\mathrm{C}-6 \mathrm{a}) \mathrm{ppm}$, indicating the connectivity of partial structures $\mathrm{A}$ and $\mathrm{B}$. The methyl protons at $\delta_{\mathrm{H}} 1.25\left(14-\mathrm{H}_{3}\right) \mathrm{ppm}$ correlated with four carbons at $\delta_{\mathrm{C}} 154.5(\mathrm{C}-6 \mathrm{a}), 46.6(\mathrm{C}-9), 45.9$ (C-9a) and $45.6(\mathrm{C}-10)$ showing the connectivity of partial structures $\mathrm{B}$ and $\mathrm{C}$. The methyl protons at
$\delta_{\mathrm{H}} 1.33\left(12-\mathrm{H}_{3}\right) \mathrm{ppm}$ showed cross peaks with the carbon signals at $\delta_{\mathrm{C}} 58.0(\mathrm{C}-3 \mathrm{a}), 78.4(\mathrm{C}-4)$ and 42.2 (C-5) ppm indicating the connectivity of partial structures $\mathrm{A}$ and $\mathrm{C}$. Correlation between the methylene protons at $\delta_{\mathrm{H}} 3.55,3.66\left(11-\mathrm{H}_{2}\right) \mathrm{ppm}$ and the carbon at $\delta_{\mathrm{c}} 35.8(\mathrm{C}-10 \mathrm{a}) \mathrm{ppm}$ were also observed.

From the above results, the structure of cyclooctatin was determined to be $1,2,3,3 \mathrm{a}, 4,5,7,8,9$,$9 \mathrm{a}, 10,10 \mathrm{a}$-dodecahydro-3,4-dihydroxy-1-hydroxy-

Fig. 1. Structure of cyclooctatin.

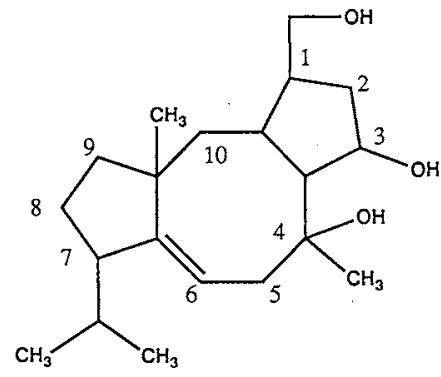

Table $1 .{ }^{13} \mathrm{C}$ and ${ }^{1} \mathrm{H}$ NMR data of cyclooctatin in $\mathrm{CD}_{3} \mathrm{OD}$.

\begin{tabular}{|c|c|c|}
\hline Carbon & $\begin{array}{c}\delta_{\mathrm{C}} \mathrm{ppm} \\
(100 \mathrm{MHz})\end{array}$ & $\begin{array}{c}\delta_{\mathrm{H}} \text { ppm } \\
(J \text { in } \mathrm{Hz}, 400 \mathrm{MHz})\end{array}$ \\
\hline 1 & 44.9 (d) & $2.61(\mathrm{~m})$ \\
\hline 2 & $39.7(t)$ & $\begin{array}{l}1.38(\mathrm{dt}, 3.4,12.6) \\
1.71(\mathrm{br} \mathrm{dd}, 12.6,5.0)\end{array}$ \\
\hline 3 & 75.7 (d) & 4.44 (br dd, $3.4,5.0)$ \\
\hline $3 a$ & $58.0(\mathrm{~d})$ & $1.97(\mathrm{t}, 5.0)$ \\
\hline 4 & $78.4(\mathrm{~s})$ & \\
\hline 5 & $42.2(t)$ & $\begin{array}{l}1.91(\mathrm{dd}, 12.8,7.4), \\
2.74(\text { br t }, 11.6)\end{array}$ \\
\hline 6 & $119.1(\mathrm{~d})$ & $5.28(\mathrm{ddd}, 10.8,7.4,2.2)$ \\
\hline $6 a$ & $154.5(\mathrm{~s})$ & \\
\hline 7 & 55.1 (d) & $2.30(\mathrm{~m})$ \\
\hline 8 & $24.3(\mathrm{t})$ & $\begin{array}{l}1.38(\mathrm{~m}) \\
1.56(\mathrm{~m})\end{array}$ \\
\hline 9 & $46.6(t)$ & $\begin{array}{l}1.42(\mathrm{~m}) \\
1.59(\mathrm{~m})\end{array}$ \\
\hline $9 \mathrm{a}$ & 45.9 (s) & \\
\hline 10 & $45.6(t)$ & $\begin{array}{l}1.20(\mathrm{t}, 12.8) \\
1.68(\mathrm{br} \mathrm{d}, 12.8)\end{array}$ \\
\hline $10 \mathrm{a}$ & $35.8(d)$ & $2.56(\mathrm{~m})$ \\
\hline 11 & $63.4(\mathrm{t})$ & $\begin{array}{l}3.55(\mathrm{dd}, 10.8,6.8) \\
3.66(\mathrm{dd}, 10.8,7.4)\end{array}$ \\
\hline 12 & $26.7(q)$ & 1.33 (br s) \\
\hline 13 & 30.2 (d) & $1.83(\mathrm{~m})$ \\
\hline 14 & $25.2(\mathrm{q})$ & $1.25(\mathrm{~s})$ \\
\hline 15 & $17.8(q)$ & $0.79(\mathrm{~d}, 6.6)$ \\
\hline 16 & $22.5(\mathrm{q})$ & $0.96(\mathrm{~d}, 6.6)$ \\
\hline
\end{tabular}


Fig. 2. Partial structures of cyclooctatin.

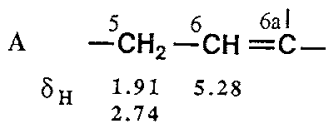

$$
\begin{aligned}
& \begin{array}{llll}
\delta_{\mathrm{C}} & 42.2 & 119.1 & 154.5
\end{array}
\end{aligned}
$$

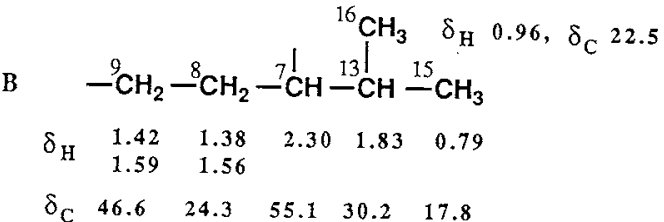$$
\text { C } \quad \stackrel{10}{-} \mathrm{CH}_{2} \stackrel{10 \mathrm{a}}{\mathrm{C}} \mathrm{CH} \stackrel{3 \mathrm{a}}{-} \mathrm{C} \mathrm{H}-\stackrel{3}{\mathrm{C}} \mathrm{CH}-{ }^{2} \mathrm{CH}_{2}-\stackrel{1}{\mathrm{C}} \mathrm{CH}-{ }^{11} \mathrm{CH}_{2}-
$$$$
\begin{array}{lccccccc}
\delta_{\mathrm{H}} & 1.20 & 2.56 & 1.97 & 4.44 & 1.38 & 2.61 & 3.55 \\
\delta_{\mathrm{C}} & 45.6 & 35.8 & 58.0 & 75.7 & 39.7 & 44.9 & 63.4
\end{array}
$$

Fig. 3. The key ${ }^{1} \mathrm{H}^{13} \mathrm{C}$ correlation by HMBC experiment.

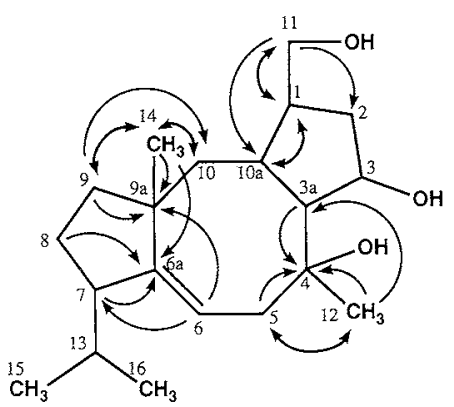

methyl-4,9a-dimethyl-7-(1-methylethyl)-dicyclopenta $[a, d]$ cyclooctene. The absolute configuration remains to be determined.

Cyclooctatin is closely related to ophiobolins (A, $B, C, D, F, G$ and $H)^{2)}$ and fusicoccin $A^{3)}$. Although these metabolites belong to the class of sesterterpenoid, only cyclooctatin is a diterpenoid compound. Moreover, cyclooctatin is produced by actinomycetes and the others are produced by fungi. Terpentecin ${ }^{4)}$, a diterpenoid antibiotic from actinomycetes, is not so similar to cyclooctatin.

\section{TAKAYUKu AOYAMA ${ }^{\dagger}$ HIROSHI NAGANAWA ${ }^{\dagger}$ YASUHIKO MURAOKA ${ }^{\dagger}$ TAKAAKI AOYAGI ${ }^{\dagger, \dagger} \dagger^{\dagger}$ TOMIO TAKEUCHI ${ }^{\dagger}$}

\author{
Institute of Microbial Chemistry \\ 3-14-23 Kamiosaki, Shinagawa-ku, \\ Tokyo 141, Japan \\ ${ }^{\dagger \dagger}$ Showa College of Pharmaceutical Sciences \\ Machida-city, Tokyo 194, Japan
}

(Received May 13, 1992)

\section{References}

1) Aoyagi, T.; T. Aoyama, F. Konima, S. Hattori, Y. Honma, M. Hamada \& T. Takeuchi: Cyclooctatin, a new inhibitor of lysophospholipase, produced by Streptomyces melanosporofaciens MI614-43F2. Taxonomy, production, isolation, physico-chemical properties and biological activities. J. Antibiotics 45: $1587 \sim 1591,1992$

2) Cutler, H. G.; F. G. Crumley, R. H. Cox, J. P. Springer, R. F. Arrendale, R. J. Cole \& P. D. ColE: Ophiobolins $\mathrm{G}$ and $\mathrm{H}$ : New fungal metabolites from a novel source, Aspergillus ustus. J. Agric. Food Chem. 32: $778 \sim 782,1984$

3) Ballio, A.; M. Brufani, C. G. Casinovi, S. Cerrini, W. Fedeli, R. Pellicciari, B. Santurbano \& A. VACIAGO: The structure of fucisoccin A. Experientia 24: $631 \sim 635,1968$

4) Isshiki, K.; T. Tamamura, Y. Takahashi, T. SaWA, H. Naganawa, T. Takeuchi \& H. Umezawa: The structure of a new antibiotic, terpentecin. J. Antibiotics 38: 1819 1821, 1985 